\title{
Non-conventional feed additives
}

\author{
A. Piva \\ Department of Veterinary Morphophysiology and Animal Production, \\ University of Bologne \\ Via Tolara Di Spora 50, 40064 Ozzano, Italy
}

\begin{abstract}
It is important that alternatives to antimicrobials for animal growth promotion are found, raising the need for a deeper understanding of the microflora physiology and interaction with the host. In this perspective, non-conventional feed additives do not have to dramatically alter the activity of the intestinal microflora, but rather have to modulate the ecosystem in which potentially toxic compounds are produced, and improve the balance of beneficial bacteria showing interesting and welldefined phenotypes. Dietary characteristics, environmental hygiene, and the risk of inducing a resistant response should all be carefully considered when designing a long-term strategy. The roles of organic acids, non-digestible oligosaccharides and natural antimicrobials are discussed.
\end{abstract}

KEY WORDS: additives, non-digestible oligosaccharides, bacteriocin, synbiotics, intestine, pigs

\section{INTRODUCTION}

The relationships between host and microflora have long been under investigation. Pasteur in 1885 appears to have been the first to question whether the wellbeing of the host could be maintained in the absence of bacteria. Germ-free mammals were found to have growth rates in the upper range of normal or even in excess of those seen under conventional conditions. The antibacterial action of sulphonamides, discovered in 1935, led Martin (1942) to interpret increased growth in rats fed $1 \mathrm{mg}$ of sulphonamide as counteraction of a disease process. Moore et al. (1946), in a study with broilers, made the important suggestion that gastrointestinal bacteria could produce toxin(s) and thereby depress growth in the absence of clinically apparent disease, or that the birds without antibiotic treatment were harbouring organisms capable of rendering unavailable certain essential growth factors. Following antibiotic supplementation, improved growth performances were 
described in chicks (Whitehill et al., 1950), turkeys (Stokstad and Jukes, 1950), pigs (Jukes et al., 1950) and ruminants (Braude et al., 1953; Jukes and Williams, 1953; Stokstad, 1954; Francois, 1962; Jukes, 1971). Thereafter, the use of antibiotics in feeds became widespread and generally the response to antibiotic feeding was greater in younger, stressed animals (Hays, 1969), and was influenced by environmental conditions (Visek, 1978).

In 1969, the Swann Committee of the United Kingdom concluded that antibiotics used in human chemotherapy or those that promote cross-resistance should not be used as growth promoters in animals. In 1985 a Swedish law was issued, proscribing that feed containing antibiotics or other chemotherapeutic substances could be sold or used only via veterinary prescription on a case-by-case basis. The legislation, applying to all livestock species, came into force on January 1, 1986, and has remained unchanged since. EU derogation allowing Sweden to operate this system expires at the end of 1998. The magnitude of antibiotic consumption is quite impressive and generates ever increasing concern. In Denmark in 1994, 24 $\mathrm{kg}$ of the glycopeptide vancomycin were used for human therapy, whereas 24,000 $\mathrm{kg}$ of the similar glycopeptide avoparcin were used in animal feeds (Witte, 1998). Vancomycin and avoparcin have the same mode of action; resistance to one can confer resistance to the other. Although the EU banned the use of avoparcin for animal feed in 1997, tylosin and virginciamycin are still in use, and both drugs can induce cross- resistance to antibiotics used in human therapy (Jacobs, 1997; Witte, 1998). It has been shown that some antibiotic resistant strains of Escherichia coli have evolved compensatory mutations that preclude reversion to the sensitive state (even without selective pressure), making the scenario of antibiotic withdrawal more urgent and dramatic (Morell, 1997).

The World Health Organization has recently proposed that there should be a reduction in the global selective pressure on antibiotics, by reducing or eliminating their use other than in human medicine (Williams and Heymann, 1998).

The objective of this study is to explore non-conventional solutions to the use of antibiotics as growth promoters.

\section{MODULATION OF GUT BACTERIAL METABOLISM}

The establishment of a microflora begins at birth and progresses through a series of colonizations involving more than 400 bacterial species (Bry et al., 1996). These colonizations result in a metabolically active entity in which anaerobes predominate. A specific region of the duodenal-colonic axis of the intestine will at any given time contain resident (autochthonous) bacterial species and a variable set of transient (allochthonous) species that temporarily occupy a functionally "cmpty" niche (Bry et al., 1996). A considerable number of factors influence the gas- 
trointestinal microflora, ranging from diet composition, to host mediated factors ( $\mathrm{pH}$, secretions, motility, tissue exudate, etc.), to microbial factors (adhesion, motility, nutritional flexibility, spores, capsules, enzymes, antimicrobial components, generation time) and microbial interactions (synergy and antagonism).

\section{Organic acids}

The use of organic acids as feed additives for monogastrics produces a variable outcome (Krause et al., 1994), possibly due to differences in the combination of the organic acids, the diet composition, or the buffering capacity, but nevertheless it has become a common practice.

The growth-promoting effect(s) of organic acids has been widely investigated, but very little is known about the mode of action of organic acids in the weaned pig relative to physiological or antimicrobial effects.

While travelling through the gastrointestinal tract neutralophiles, like E. coli, Salmonella typhimurium or Shigella flexneri, must endure the extreme, low $\mathrm{pH}$ of the stomach as well as the short chain fatty acids (SCFA) present in the intestine and faeces. These bacteria are, therefore, exposed to acid stress, which can be described as the combined biological effect of low $\mathrm{pH}$ and weak (organic) acids present in the environment. Weak acids, including SCFA produced as result of intestinal fermentation, can diffuse across the cell membrane in the uncharged, protonated form and dissociate inside the cell, lowering internal $\mathrm{pH}\left(\mathrm{pH}_{\mathrm{i}}\right)$ (Bearso et al., 1997). The lower the external $\mathrm{pH}\left(\mathrm{pH}_{0}\right)$, the more undissociated weak acid will be available (based upon $\mathrm{pK}_{\mathrm{a}}$ values) to cross the membrane and affect $\mathrm{pH}_{\mathrm{i}}$. To overcome the lowering of $\mathrm{pH}_{i}$, several amino acid decarboxylases are induced; these elevate the $\mathrm{pH}_{i}$ by consuming a proton during decarboxylation and they then exchange the decarboxylation end-product for a new substrate via a membrane-bound antiporter (Bearso et al., 1997). One example is lysine decarboxylase (CadA) coupled with the lysine-cadaverine antiporter $(\mathrm{CadB})$ of $S$. typhimurium. The CadA decarboxylates intracellular lysine to cadaverine and consumes a proton in the process. Cadaverine is then exchanged for fresh lysine from the surrounding environment via the $\mathrm{CadB}$ antiporter (Park et al., 1996). Similar inducible systems, with arginine and glutamate decarboxylases, have been described for $E$. coli (Lin et al., 1995).

Guilfoyle and Hirshfield (1996) demonstrated that organic acids could induce acid resistance. For example, $E$. coli adapted with $0.1 \%$ butyrate or propionate at $\mathrm{pH}_{0} 6.5$ survived a 30 -min challenge at $\mathrm{pH}_{0} 3.5$ some 50 - to 200 -fold better than cells adapted at $\mathrm{pH}_{0} 6.5$ in the absence of the weak acid. These investigators also suggested that arginine and lysine decarboxylases might contribute to survival in organic acids.

Cells undergoing acid shock in the stomach are well prepared to endure the environmental stresses subsequently confronted in the intestine (Bearso et al., 1997). 
The acid habituation is worse if organic acids are added to the diet in the free form. The use of microencapsulated organic acids, that by-pass the stomach and exert an antimicrobial activity in the upper gut, represents one strategy to overcome this problem. Additionally, acid shock induces cross-resistance to a variety of stresses and is also correlated to the pathogenesis of enteric bacteria (Bearso et al., 1997). The choice of the appropriate acid mixture relative to both $\mathrm{pK}_{\mathrm{a}}$ and the acid delivery system is critical for its efficacy.

\section{Non-digestible oligosaccharides}

Dietary modulation of the colonic microbiota using prebiotics is a fairly recent strategy (Gibson and Robertfroid, 1995). Several investigators have reported that certain non-digestible oligosaccharides (NDO) specifically promote the growth of bifidobacteria (Tanaka et al., 1983; Mutai et al., 1984; Hidaka et al., 1986; Hayakawa et al., 1990). Considerable efforts have been directed to implementing and optimizing carbohydrates, with a degree of polymerization (DP) of from 2 to 20

TABLE 1

Fermentation of NDO by selected intestinal bacteria* (Hartemink and Rombouts, 1997)

\begin{tabular}{llllllllll}
\hline Bacterial group/species & FOS & INU & TOS & GLL & IMO & RAF & LAT & LAC PHGG \\
\hline Bacteroides distasonis & + & + & + & + & + &,+- & + & + & - \\
B. fragilis & + & + & + & + & + &,+- & + & + & - \\
B. ovatus & + & + & + & - & + &,+- & + & & + \\
B. thetaiotaomicron & + & + & + & & + &,+- & & + & - \\
B. vulgatus & + & + & + & + & + &,+- & + & & - \\
Bifidobacterium spp. & + & + & + & + & + & + & + &,+- & - \\
Clostrium butyricum & & - & - & - & - & + & + & + & + \\
Cl. clostridioforme &,+- & - & & - & - &,+- & - & + & - \\
Cl. perfringens &,+- &,-+ &,-+ & - & + &,+- & + & + & - \\
Cl. ramosum & + & + & & - & + &,+- & + & + & - \\
Escherichia coli &,-+ & - & + & - & - & - &,+- & - & - \\
Eubacterium lentum & - & - & & - & - & - & - & & \\
Eu. Limosum & - & - & - & - & - & - & - & & \\
Fusobacterium necrophorum & - & - & - & - & - & - & - & & - \\
Lactobacillus acidophilus-group &,+- & + & + & + &,+- & - & + & + & - \\
Lb. Casei &,+- & + & + & - & - & - & + & + & - \\
Megasphaera elsdenii & - & - & - & - & & - & - & - & - \\
Mitsuokella multiacidus &,+- & + & & - & + & + & + & & - \\
R. productus & - & & & - & - &,+- & + & & \\
Veillonella parvula & - & - & - & - & - & - & - & - & \\
\hline
\end{tabular}

' FOS: fructo-oligosaccharides, INU: inulin, TOS: trans-galactosyl-oligosaccharides, GLL: 4'-galactosyl-lactose, IMO: isomalto-oligosaccharides, RAF: raffinose, LAT: lactulose, LAC: lactitol, PHGG: partially hydrolized guar gum 
sugar units, as prebiotics for foods and feeds. Linear oligosaccharides are fermented to a higher extent and by a greater number of different bacterial species than are branched oligosaccharides (Van Laere et al., 1997). In addition, the sugar building blocks (units) and the degree of polymerization influence the pathway of fermentation. Bifidobacteria initially utilize oligosaccharides with a low DP, whereas Bacteroides ferment initially oligosaccharides with a higher DP (Van Laere et al., 1997). Several bacteria are capable of producing a variety of glycosidases that are mainly induced upon growth on oligosaccharides.

Depending on the structure, degradation of NDO can occur at the terminal ileum, in the caecum or in the colon. Many NDO display bifidogenic properties and increase the bifidobacteria count in different sections of the gastrointestinal tract and/or faeces. To exert this effect, it is desirable that NDO are fermented exclusively or preferentially by bifidobacteria and not by other intestinal bacteria (Hartemink and Rombouts, 1997). As bifidobacteria do not produce hydrogen or carbon dioxide (Holdeman and Moore, 1977), fermentation by bifidobacteria does not result in gastrointestinal bloating and pain. Unfortunately, gas production is often described as a drawback of the use of NDOs. This suggests that even though bifidobacteria appear to be able to ferment most NDOs, it is likely that NDOs are also degraded by intestinal gas $\left(\mathrm{H}_{2}, \mathrm{CO}_{2}\right.$, and $\left.\mathrm{CH}_{4}\right)$ producing bacteria, namely clostridia, eubacteria, and anaerobic cocci (Table 1).

\section{In vitro studies}

We have developed an in vitro fermentation system that allows an appropriate comparison of the effect of additives on intestinal microflora in the upper and lower gut (Piva et al., 1996a). The system allows us to screen and narrow the number of possible alternatives to be evaluated in vivo.

Preliminary experiments revealed that the effects of antibiotics, organic acids and NDO on the intestinal microflora are dramatically different. In a comparative trial with antibiotics (carbadox: $50 \mathrm{ppm}$; flavophospholipol $10 \mathrm{ppm}$ ), commercially available acidifier mixtures (acidifier 1 used at $0.3 \%$ and containing $10 \%$ ortophosphoric, $10 \%$ citric, $10 \%$ malic, $20 \%$ fumaric acids; acidifier 2 used at $0.1 \%$ and containing $35 \%$ ortophosphoric, $6 \%$ fumaric, $2 \%$ citric acids), and NDOs (NDO 1 used at $0.5 \%$ containing $37.3 \%$ oligosaccharides, $2.4 \%$ ortophosphoric, $2.4 \%$ citric, $2.4 \%$ malic, and $4.8 \%$ fumaric acids; and NDO 2 used at $0.5 \%$ containing $37.3 \%$ oligosaccharides), small intestine ammonia production rate was reduced by carbadox $(-78 \%)$, flavophospholipol (-27\%), NDO $1(-17 \%)$ and NDO $2(-15 \%)$ while in the caecum acidifier 1 and 2 resulted in lower ammonia production rates, by 32 and $27 \%$ respectively, compared to antibiotic treated vessels (Piva, unpublished results). Concomitantly, gas production was depressed when using antibiotics (Figure 1), whereas NDOs cnhanced it, depending on the type oligosaccha- 


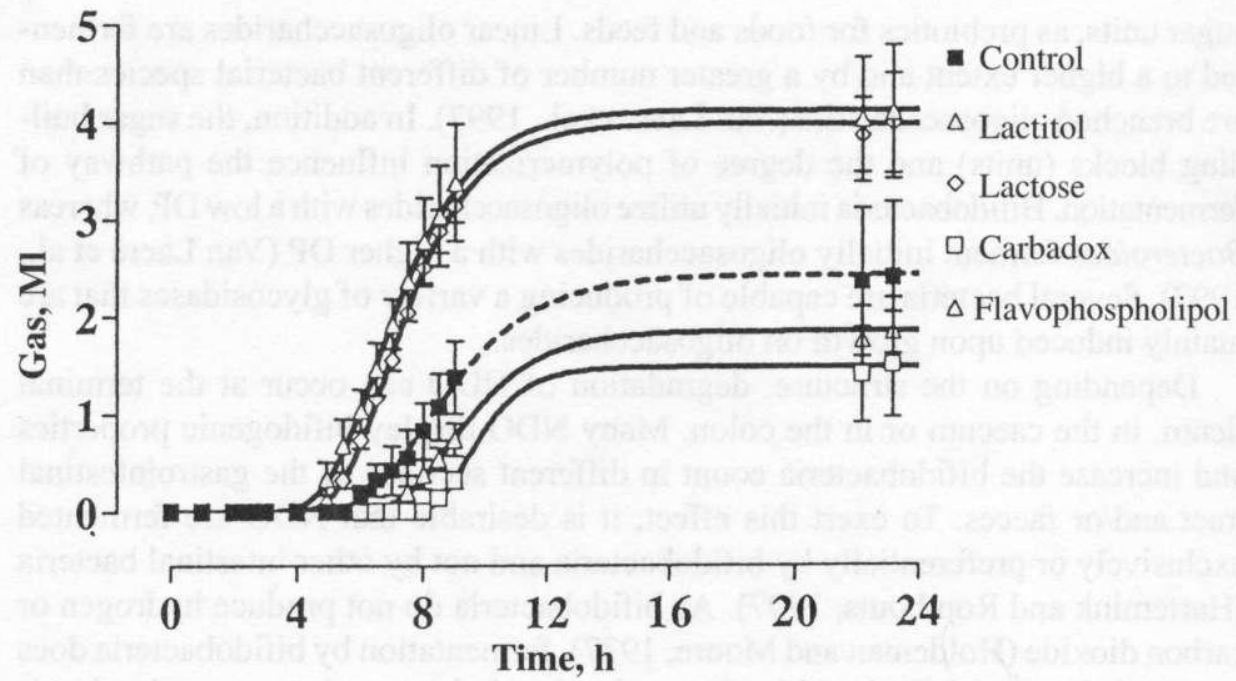

Figure 1. Gas production from in vitro incubation of swine caecal microflora and a low predigested diet (control) with the addition of carbadox (50 ppm), flavophospholipol (10 ppm), lactitol or lactose $(3 \mathrm{mmol} / \mathrm{L})$. Bars indicate SD

ride structure (Hartemink and Rombouts, 1997) and the type of basal diet fed to the animals (Piva et al., 1996b).

Increasing interest is being generated in the use for animal nutrition of certain sugars, such as lactulose and lactitol (Nousiainen and Setšlš, 1992), usually employed in the treatment of human portosystemic encephalopathy (Bircher et al., 1966). As the latter disaccharide is not normally absorbed in the small intestine (Dharmaraj et al., 1987) it reaches the lower gut (Nousiainen and Setšlš, 1992), where it is fermented. The presence of this sugar alcohol in the caecum and colon prevents the deamination of amino acids and reduces ammonia concentration and its absorption by the host (Jensen, 1993); it enhances SCFA and gas production by pig caecal microflora to a greater extent when fermenting low rather than high fibre diets (Piva et al., 1996b).

Recent investigations have revealed that lactitol is effective in reducing caecal microbial production rate of indole and 3-methylindole (skatole) by 20 and $22 \%$ and by 10 and $48 \%$ respectively, when added to LF or HF fed fermentors (Piva et al., 1997a). Since skatole can be responsible for pulmonary oedema and emphysema in cattle, horses and rats, and since skatole contributes to the unpleasant taste and odour in cooked pork, control of the intestinal production of skatole has practical importance in animal farming (Henry, 1995). Short-chain fatty acid proportions are shifted to higher levels of propionic acid (Houdijk et al., 1997) and net energy yields in the caecum can be increased by 40 to $70 \%$ in NDO fed fermentors (Piva et al., 1996b). 
Potential drawbacks could result from the high gas production in terms of abdominal pain and from exposure of the terminal ileum to high levels of SCFA, which could inhibit gastric motility (referred to as the "ileal brake"; Cuche and Malbert, 1997). This may temporarily affect the voluntary intake, as reportedly occurred in some cases during adaptation to NDO (Houdijk et al., 1997; Mul, 1997).

It is becoming evident that appropriately selecting and pairing NDOs and probiotic strains, that show an interesting and definite phenotype (expressed in the upper or lower intestine and not only in a synthetic medium), and posses the metabolic capability of fermenting the supplied NDO at a competitive rate compared to the indigenous microflora, is likely to be a successful strategy in controlling the intestinal ecosystem.

Since the mode of action of some probiotics can be related to the production of antimicrobial substances, we investigated the effect of pediocin A (a bacteriocin produced by Pediococcus pentosaceus FBB61; Piva and Headon, 1994) in in vitro caecal fermentation (Piva et al., 1995). The antimicrobial spectrum of this bacteriocin includes several strains of Listeria monocytogenes (Spelhaug and Harlander, 1989), Lactobacillus and Staphylococcus spp. and clostridia (Piva and Headon, 1994), but it had no direct effect against any gram-negative bacteria tested.

To verify the expression and activity of this antimicrobial phenotype in the caecum, . pentosaceus FBB61, pediocin A producer (bact), and its isogenic mutant (bac-) P. pentosaceus FBB61-2 were added to the fermentation vessels (Piva et al., 1995). Pediocin A did not alter the normal activity of caccal microflora. Nevertheless, the presence of pediocin A producer strain reduced proteolysis compared to the mutant strain, as indicated by the ammonia concentrations $(\mathbf{P}<0.05)$, and the molar proportions of isobutyric and isovaleric acids $(\mathrm{P}<0.05)$. The expression of pediocin A and its immunogenicity are linked to the pMD136 plasmid (Daeschel and Klaenhammer, 1985). Since this plasmid does not encode for other phenotypes, such as carbohydrate fermentation or antibiotic resistance (Piva et al., 1995), it was concluded that pediocin A was solely responsible for the differences in the fermentation and chemical parameters observed between strains FBB61 and FBB61-2.

To further eliminate any possible interference due to the growth of the added pediococcal strains to the fermentation vessels, partially purified pediocin A was directly added to the caccal fermentation to evaluate possible metabolic responses due to antimicrobial activity, especially against lactic acid bacteria and clostridia. Culture supcrnatant of $P$. pentosaceus FBB61 (bac + ) and its isogenic mutant FBB61-2 (bac-) were fractionated using an anion exchange column leading to a 34 -fold increase in the specific activity of the bacteriocin, with $61 \%$ activity recovered. The eluted antimicrobial bac + fraction and the corresponding fraction from bac- mutant were studied in an in vitro caecal fermentation. The pig caecal innoculum was incubated for $48 \mathrm{~h}$ in 3 vessels per treatment given a standard 


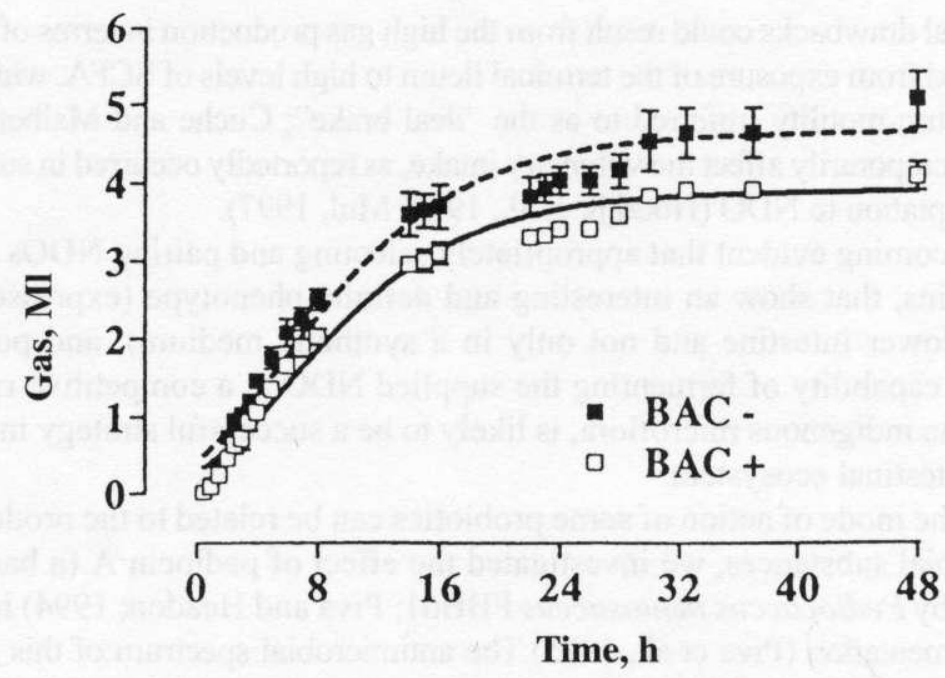

Figure 2. Partially purified pediocin A lowered maximum gas production by $15 \%$ for bac + vs bacvessels (3.9 vs $4.6 ; \mathrm{P}=0.08$ )

predigested diet (control) or the same diet added with the active fraction (bac + : 128 activity units $(\mathrm{AU}) / \mathrm{mL}$ ) or with the non-active fraction (bac-). Ammonia, SCFA, tryptophan, tryptamine, indole-3-acetic acid, indole-3-propionic acid, indole, and skatole were analyzed in triplicate per treatment at $0,4,8,16,24,30$ and $48 \mathrm{~h}$. The molar proportion of propionic acid was higher $(\mathrm{P}<0.05)$ in bac + treated vessels, whereas n-butyric acid was lower $(\mathrm{P}<0.05)$ throughout the $48 \mathrm{~h}$. Pediocin A reduced ammonia production rate in the first $4 \mathrm{~h}$ by $33 \%(1.82 \mathrm{vs} 2.72 \mathrm{mmol} / \mathrm{L} \mathrm{h}$; $\mathrm{P}<0.05$ ) with no further difference until $48 \mathrm{~h}$. Gompertz bacterial growth model was fitted to the gas production data; it showed a $15 \%$ lower maximum gas production for bac + vs bac- vessels ( 3.9 vs $4.6 ; \mathrm{P}=0.08$; Figure 2 ). Interestingly, clostridia are one of the most gas-producing group of bacteria in the intestine (Hartemink and Rombouts, 1997) and they are considered to be sensitive to pediocin A.

Pediocin A modified tryptophan degradation by caecal microflora, causing a constant reduction of skatole concentration by $21.5 \%(\mathrm{P}<0.05)$ between 16 and $48 \mathrm{~h}$. Pediocin A also caused an unexpected increase (53\%) in indole concentration between 4 and $48 \mathrm{~h}(\mathrm{P}<0.05$; Figure 3$)$, but no other effect could be observed on any indolic compound analyzed. Interestingly, many strains of intestinal bacteria can produce indole from L-tryptophan, whereas only some strains of the Clostridium and Lactobacillus genera are reported to produce skatole (Jensen et al., 1995) and show sensitivity to pediocin A (Piva and Headon, 1994).

These results indicate that pediocin A influences the intestinal ecology, selecting a microflora with lower gas production, higher propionic acid and indole yield, and lower skatole production (Piva et al., 1997b). 

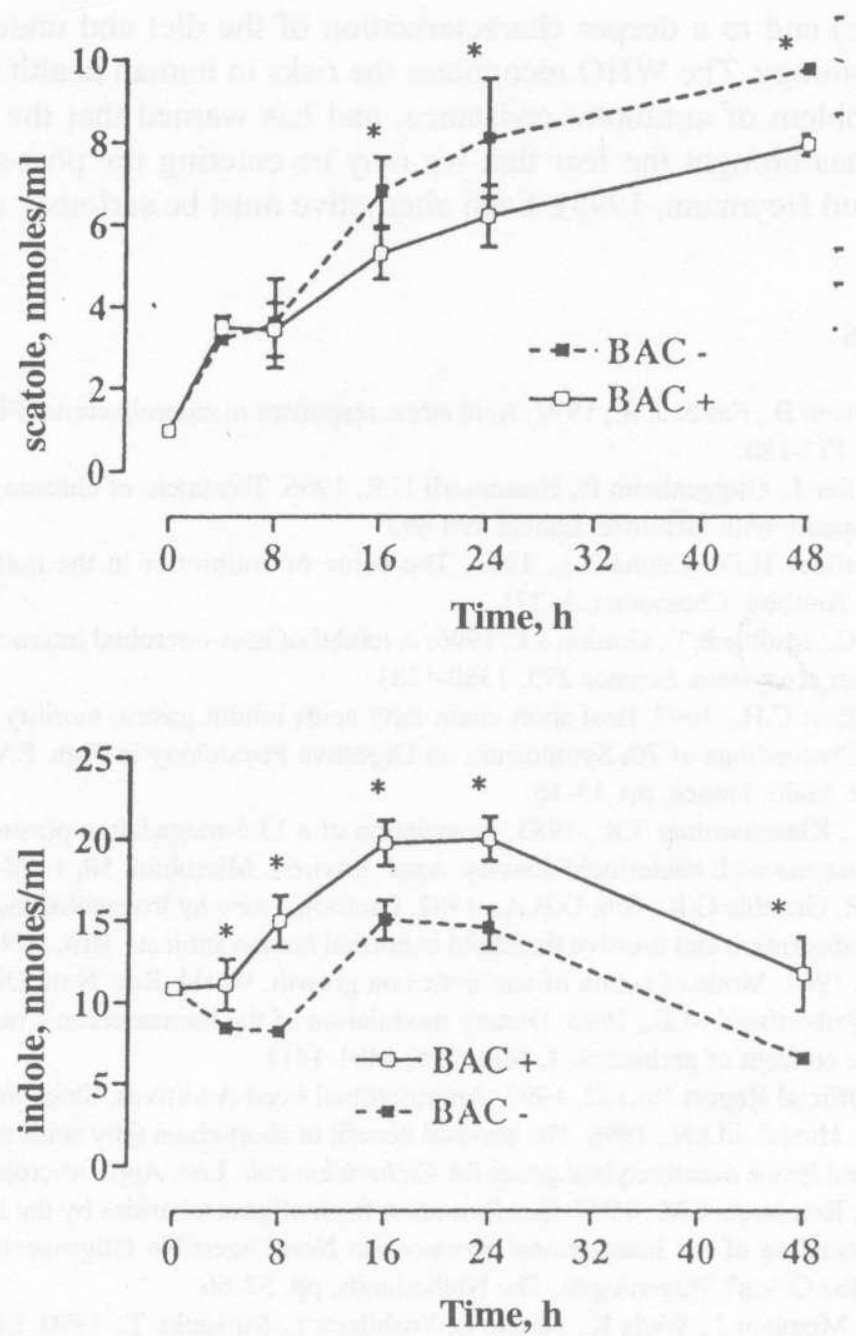

Figure 3. Partially purified pediocin A effect on in vitro caecal skatole and indole concentrations

\section{CONCLUSIONS}

The need to contain the spread of antibiotic resistance requires that alternative strategies must be carefully examined. Promising results can be obtained using synbiotics; the appropriate match of NDO together with probiotic microbes which have been carefully selected for specificity, strong competition for the supplied NDO and capable of showing definitive phenotypes in the intestine. This approach can be successful if coupled to procedural modification in animal management 
(i.c. hygiene) and to a deeper characterization of the diet and understanding of animal physiology. The WHO recognizes the risks to human health posed by the growing problem of antibiotic resistance, and has warned that the lack of new antibiotics has brought the fear that we may be entering the post-antibiotic era (Williams and Heymann, 1998). Each alternative must be seriously considered.

\section{REFERFNCES}

Bearso S., Bearson B., Foster J.W., 1997. Acid stress responses in enterobacteria. FEMS Microbiol. Lett. $147,173-180$

Bircher J., Muller J., Guggenheim P., Haemmerli U.P., 1966. Treatment of chronic portal-systemic encephalopathy with lactulose. Lancet 890-893

Braude R., Wallace H.D., Cunha T.J., 1953. The value of antibiotics in the nutrition of swine. A review. Antibiot. Chemother. 3, 271

Bry L., Falk P.G., Midtvedt T., Gordon J.I., 1996. A model of host-microbial interactions in an open mammalian ccosystem. Science $273,1380-1383$

Cuche G., Malbert C.H., 1997. Ileal short chain fatty acids inhibit gastric motility via an humoral pathway. Procedings of 7 th Symposium on Digestive Physiology in Pigs. FAAP Publication No. 88. St. Malo, France, pp. 13-16

Daeschel M.A., Klaenhammer T.R., 1985. Association of a 13.6-megadalton plasmid in Pediococcus pentosaceus with bacteriocin activity. Appl. Environ. Microbiol. 50, 1538-1541

Dharmaraj H.P., Grimble G.K., Silk D.B.A., 1987. Lactitol, a new hydrogenated lactose derivative: intestinal absorption and laxative threshold in normal human subjects. Brit. J. Nutr. 57, 195-199

Francois A.C., 1962. Mode of action of antibiotics on growth. World. Rev. Nutr. Diet. 3, 21

Gibson G.R., Robertfroid M.B., 1995. Dietary modulation of the human colonic microbiota: introducing the concept of prebiotics. J. Nutr. 125, 1401-1412

Government Official Report No.132, 1997. Antimicrobial Feed Additives. Stockholm, Sweden

Guilfoylc D.E., Hirshfield I.N., 1996. The survival benefit of short-chain fatty acids and the inducible arginine and lysine decarboxylase genes for Escherichia coli. Letl. Appl. Microbiol. 22, 1-4

Hartemink R., Rombouts F.M., 1997. Gas formation from oligosaccharides by the intestinal microflora. Proceeding of the International Symposium Non-Digestible Oligosaccharides: Healthy Food for the Colon? Wageningen, The Netherlands, pp. 57-66

Hayakawa K., Mizutani J., Wada K., Masai T., Yoshihara I., Mitsuoka T., 1990. Effect of soybean oligosaccharides on human faecal flora. Microb. Ecol. Health Disease 3, 293-303

Hays V.W., 1969. Biological basis for the use of antibiotics in livestock production. The use of drugs in animal feeds. National Academy of Sciences, Publ. 1697

Henry Y., 1995. Effects of dietary tryptophan deficiency in finishing pigs, according to age or weight at slaughter on live weight gain. Livest. Prod. Sci. 41, 63-76

Hidaka H., Eida T., Takizawa T., Tokunaga T., Tashiro Y., 1986. Effects of fructooligosaccharides on intestinal flora and human health. Bifidobact. Microflora 5, 37-50

Holdeman L.V., Moore W.E.C., 1977. Anaerobe Lab Manual Virginia Polytechnic Institute and State University. 4th Edition. Blacksburg, Virginia, USA

Houdijk J.G.M., Hartemink R., Van Laere K.M.J., Williams B.A., 1997. Fructooligosaccharides and transgalactooligosaccharides in weaner pigs dict. Proceeding of the International Symposium Non-Digestible Oligosaccharides: Healthy Food for the Colon? Wageningen, The Netherlands, pp. $69-78$ 
Jacobs C., 1997. Life in the balance: cell walls and antibiotic resistance. Science 278, 1731-1732 Jensen B.B., 1993. The possibility of manipulating the microbial activity in the digestive tract of monogastric animals. Proceedings of 44th Meeting of European Association for Animal Production, pp. 1-20

Jensen M.T., Cox R.P., Jensen B.B., 1995. 3-methyl-indole (skatole) and indole production by mixed populations of pig fecal bacteria. Appl. Environ. Microbiol. 61, 3180-3184

Jukes T.H., 1971. The present status and background of antibiotics in the feeding of domestic animals. Ann. N.Y. Acad. Sci. 182, 362

Jukes T.H., Stockstad E.L.R., Taylor R.R., Cunha T.J., Edwards H.M., Meadows G.B., 1950. Growth promoting effect of aureomycin on pigs. Arch. Biochem. 26, 324

Jukes T.H., Williams W.L., 1953. Nutritional effects of antibiotics. Pharmacol. Rev. 5, 381

Krause D.O., Harrison P.C.. Easter R.A., 1994. Characterization of the nutritional interactions between organic acids and inorganic bases in the pig and chick. J. Anim. Sci. 72, 1257-1262

Lin J., Lee I.S., Frey J., Slonozewski J.L., Foster J.W., 1995. Comparative analysis of extreme acid survival in Salmonella typhimurium, Shigella flexneri and Escherichia coli. J. Bacteriol., 177, 4097-4104

Martin G.J., 1942. p-Aminobenzoic acid and sulfonamides in rat nutrition. Proc. Soc. Exp. Biol. Med. 51,56

Moore P.R., Evenson A., Luckey T.D., McCoy E., Elvehjem C.A., Hart E.B., 1946. Use of sulfasuxidine, streptothricin, and streptomycin in nutritional studies with the chick. J. Biol. Chem. 16, 437

Morrel V., 1997. Antibiotic resistance: road of no return. Science 278, 575-576

Mul A.J., 1997. Application of oligosaccharides in animal feed. Proceeding of the International Symposium "Non-Digestible Oligosaccharides: Healthy Food for the Colon?". Wageningen, The Netherlands, p. 106

Mutai M., Terashima T., Takahashi T., Tanaka R., Kuroda A., Ueyama S., Matsumoto K., 1984. Composition for promoting growth of bifidobacteria. United States Patent Application 166, 463, Publication No 4, 435, 389

Nousiainen J.T., Setšlš J.K., 1992. Feed for promoting the growth and intestinal function of animals. European Patent Application 91108549.6 , Publication No 0464362 Al

Park Y.K., Bearson B., Bang S.H., Bang I.S., Foster J.W., 1996. Internal pH crisis, lysine decarboxylase and the acid tolerance response of Salmonella typhimurium. Mol. Microbiol. 20, 605-611

Piva A., Headon D.R., 1994. Pediocin A, a bacteriocin produced by Pediococcus pentosaceus FBB61. Microbiology 140, 697-702

Piva A., Meola E., Panciroli A., 1995. Effect of Pediococcus pentosaceus FBB61, pediocin A producer strain, in caecal fermentations. J. Appl. Bacteriol. 78, 616-620

Piva A., Meola E., Panciroli A., Formigoni A., 1996a. In vitro intestinal fermentation and modelling. J. Anim. Sci. 74, Suppl.1, 173 (Abstract)

Piva A., Panciroli A., Meola E., Formigoni A., 1996b. Lactitol enhances short-chain fatty acid and gas production by swine cecal microflora to a greater extent when fermenting low rather than high fiber diets. J. Nutr. 126, 280-289

Piva A., Meola E., Formigoni A., Panciroli A., Bertuzzi T., Pietri A., Mordenti A., 1997a, Lactitoi controls indole and 3-methylindole production by swine cecal microflora. 7th Symposium on Digestive Physiology in Pigs. EAAP Publication No. 88. St. Malo, France, pp. 470-474

Piva A., Luchansky J.B., Pietri A., Biagi G., Meola E., Testi S., Bertuzzi T., 1997b. Role of lactic acid bacteria pediocin A on intestinal fermentation. J. Anim. Sci. 75, Suppl. 1, 200 (Abstract)

Spelhaug S.R., Harlander S.K., 1989. Inhibition of foodborne bacterial pathogens by bacteriocins from Lactococcus lactis and Pediococcus pentosaceus. J. Food. Prot. 52, 856-862

Stokstad E.L.R., 1954. Antibiotics in animal nutrition. Physiol. Rev. 34, 25 
Stokstad E.L.R., Jukes T.H., 1950. The growth promoting effect of aureomycin on turkey poults. Poultry Sci. 29, 611

Swann Committee, 1969. Report of Joint Committee on the Use of Antibiotics in Animal Husbandry and Veterinary Medicinc, Swann Committee, Her Majesty's Stationery Office, London, September 1969

Tanaka R., Takayama H., Morotomi M., 1983. Effects of administration of TOS and Bifidobacterium breve 4006 on the fecal flora. Bifidobact. Microflora 2, 17-24

Van Laere K.M.J., Bosveld M., Schols H.A., Beldman G., Voragen A.G.J., 1997. Fermentative degradation of plant cell wall derived oligosaccharides by intestinal bacteria. Procecding of the International Symposium "Non-Digestiblc Oligosaccharides: Healthy Food for the Colon?". Wageningen, The Netherlands, pp. 37-46

Visek W.J., 1978. The mode of growth promotion by antibiotics. J. Anim. Sci. 46, 1447-1469

Whitehill A.R., Oleson J.J., Hutchings B.L., 1950. Stimulatory effect of aureomycin on the growth of the chicks. Proc. Soc. Exp. Biol. Med. 74, 11

Williams R.J., Heymann D.L., 1998. Containment of antibiotic resistance. Science 279, 1153-1154 Witte W., 1998. Medical consequences of antibiotic use in agriculture. Science 279, 996-997 